\title{
Cryo-STEM Reveals Humidity-Controlled Shape Change in Silica Nanoparticles
}

\author{
Katherine A. Spoth ${ }^{1}$, Yao Sun ${ }^{2}$, Ulrich Wiesner ${ }^{2}$ and Lena F. Kourkoutis ${ }^{1,3}$ \\ 1. Department of Applied and Engineering Physics, Cornell University, Ithaca, New York, USA. \\ 2. Department of Materials Science and Engineering, Cornell University, Ithaca, New York, USA. \\ 3. Kavli Institute at Cornell for Nanoscale Science, Ithaca, New York, USA.
}

Cryo-electron microscopy allows the direct observation of cryo-immobilized structures in solution. It therefore is a powerful tool for visualizing formation processes in liquids for which resolution, contrast, or beam damage prevents real-time liquid cell TEM observation. Here, we use cryo-STEM to image at high-resolution hybrid mesoporous silica nanoparticles with structure dictated by the interaction between inorganic silica and an ordered organic template. We demonstrate that surprisingly these silica particles behave as shape change materials (SCMs). Shape changes can be induced via the presence of water, e.g. by controlling the humidity during particle drying, suggesting potential applications of these silica-based nanoparticles as humidity-responsive material.

Mesoporous silica nanomaterials show great potential for applications in a variety of fields. Single-pore particles have been investigated for drug-delivery purposes, having a sufficiently small diameter for removal by the kidneys to avoid toxic effects [1]. Larger particles with high surface area due to their interconnected pore structure are promising for energy and sensor applications [2]. While porous silica materials have been extensively studied, their formation is not well understood. Cryo-TEM allows study of these solution-based processes as demonstrated recently for assembly of monodisperse silica nanoparticles [3]. Typically, however, these materials are characterized by conventional electron microscopy in which the drying process can alter the materials' structure.

To reveal the structure of mesoporous silica nanoparticles in solution ( $80 \%$ ethanol and $20 \%$ water), we vitrified the sample by plunge freezing using a liquefied ethane/propane mixture and imaged it with cryo-STEM. Figure 1 shows the particles' regular hexagonal shape and symmetric pore structure, visible in both front and side views of single particles. The average pore spacing determined from the power spectrum of the image is $\sim 3.6 \mathrm{~nm}$. The image resolution is at least $1.8 \mathrm{~nm}$ as indicated by clear spots at that spacing. In contrast, when the sample is dried directly on the grid for traditional TEM the structure shrinks and deforms, resulting in a star-shaped cross-section.

To understand these structural modifications, we have studied the effect of the drying environment on the particle shape. Starting with the known structure imaged by cryo-STEM (Fig. 2a, d), the sample was dried in the vacuum of the microscope by warming it to room temperature, allowing the ice to sublimate during the process. Surprisingly, the particle shape remained close to hexagonal (Fig. 2e), however, its size shrank by $\sim 10 \%$, from $176 \mathrm{~nm}$ to $159 \mathrm{~nm}$ (corner to corner distance). Subsequently, the sample was stored in a humid environment and re-imaged after 12 days. The particles' shape changed significantly with some pores visible but irregular (Fig. 2c, f); in addition the size of the particle further decreased to $137 \mathrm{~nm}$. The effect of water on the particle shape was confirmed by imaging particles synthesized in pure water. These particles exhibited star-shaped cross sections even when imaged by cryo-STEM [4].

[1] K Ma et al, Chem. Mater., 25 (2013), p. 677-691.

[2] F Hoffmann et al, Angew. Chem. Int. Ed. 45 (2006), p. 3216 - 3251.

[3] C Carcouët et al, Nano Letters, 14 (2014), p. 1433-1438.

[4] This work was supported by the Cornell Center for Materials Research with funding from the NSF MRSEC program (DMR-1120296). 

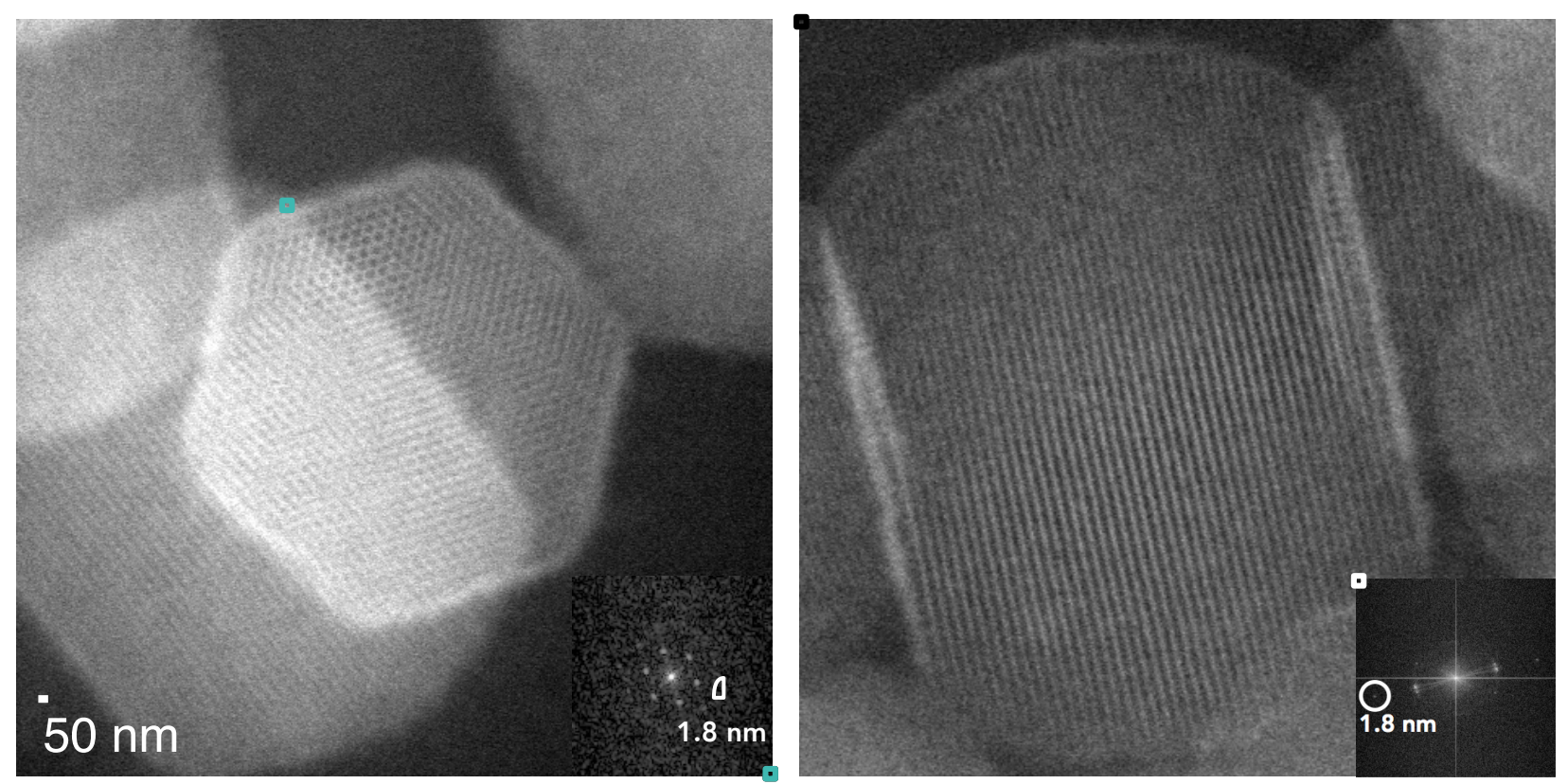

Figure 1. Cryo-STEM reveals symmetric pore structure of silica nanoparticles embedded in vitreous ice. Front (left) and side (right) orientations show average pore spacing of $\sim 3.6 \mathrm{~nm}$ confirmed by FFT with resolution better $1.8 \mathrm{~nm}$.
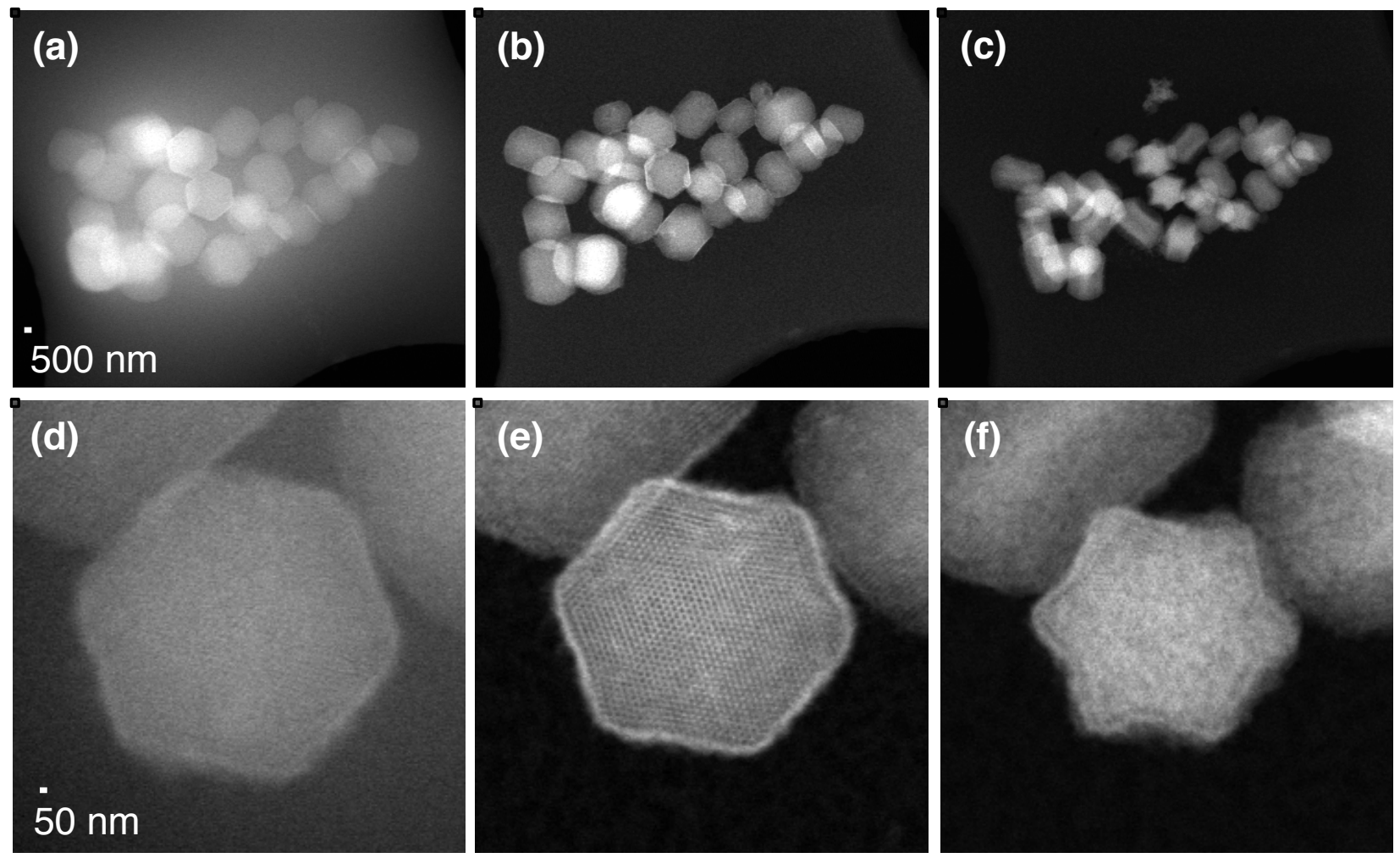

Figure 2. Shape change behavior of silica nanoparticles in response to humidity during drying process. Cryo-STEM shows regular, symmetric hexagonal structure of material $(a, d)$. After vacuum drying in the microscope column, the same particles exhibit minor changes in shape $(b, e)$. Exposing the sample to humid air causes complete deformation of the structure (c, f). A single particle shows size decrease when dried (d-f). Starting at $176 \mathrm{~nm}$ in solution, vacuum drying decreases the corner-to-corner distance to $159 \mathrm{~nm}$; further shrinkage to $137 \mathrm{~nm}$ occurs upon exposure to humidity. 